\title{
ON FRACTIONAL POINCARÉ INEQUALITIES
}

\author{
RITVA HURRI-SYRJÄNEN AND ANTTI V. VÄHÄKANGAS
}

\begin{abstract}
We show that fractional $(p, p)$-Poincaré inequalities and even fractional Sobolev-Poincaré inequalities hold for bounded John domains, and especially for bounded Lipschitz domains. We also prove sharp fractional $(1, p)$-Poincaré inequalities for $s$-John domains.
\end{abstract}

\section{INTRODUCTION}

We consider the following fractional $(q, p)$-Poincaré inequality in a bounded domain $G$ in $\mathbb{R}^{n}, n \geq 2$,

$$
\int_{G}\left|u(x)-u_{G}\right|^{q} d x \leq c\left(\int_{G} \int_{G \cap B^{n}(x, \tau \operatorname{dist}(x, \partial G))} \frac{|u(x)-u(y)|^{p}}{|x-y|^{n+\delta p}} d y d x\right)^{q / p},
$$

where $1 \leq p, q<\infty, \delta, \tau \in(0,1)$, and the constant $c$ does not depend on $u \in L^{p}(G)$. Our inequality (1.1) with $q=p$ is stronger than the fractional inequality

$$
\int_{G}\left|u(x)-u_{G}\right|^{p} d x \leq c \int_{G} \int_{G} \frac{|u(x)-u(y)|^{p}}{|x-y|^{n+\delta p}} d x d y,
$$

where on the right hand side is the commonly used seminorm on $W^{\delta, p}(G)$, [A]. Augusto C. Ponce showed that bounded Lipschitz domains support the same type of inequalities as (1.2) but with general radial weights, [P1], [P2, Theorem 1.1]. Jean Bourgain, Haïm Brezis, and Petru Mironescu found the optimal constant $c$ in (1.2) when $G$ is a cube [BBM2, Theorem 1]. An elementary proof was provided by Vladimir Maz'ya and Tatyana Shaposhnikova, [MS1], [MS2]. The relationship between the right hand side of (1.2) and the $L^{p}(G)$ integrability of the absolute value of the gradient in smooth bounded domains is considered in [BBM1].

We give sufficient geometric conditions for a bounded domain $G$ in $\mathbb{R}^{n}$ to support the fractional $(q, p)$-Poincaré inequality for $1 \leq q \leq$ $p<\infty$, Theorem 3.1. Examples of the domains which support the fractional $(p, p)$-Poincaré inequality are John domains, Theorem 4.3. The John domains include uniform domains and hence also Lipschitz

Date: August 5, 2018.

2010 Mathematics Subject Classification. 46E35 (26D10).

Key words and phrases. fractional Poincaré inequality, fractional SobolevPoincaré inequality, uniform domain, s-John domain, porous set.

A.V.V. was supported by the Academy of Finland, grants 75166001 and 1134757. 
domains. We show that John domains support the fractional Poincaré inequality (1.1) when $1<p \leq q \leq n p /(n-\delta p)$ and $p<n / \delta$, Theorem 4.10. We also study more general bounded domains, so called $s$-John domains with $s>1$. We prove fractional $(1, p)$-Poincaré inequalities for these domains, Theorem 5.1, and we show that these results are sharp, Theorem 6.9.

\section{NOTATION AND AUXILIARY RESUltS}

We assume that $G$ is a bounded domain in Euclidean $n$-space $\mathbb{R}^{n}$, $n \geq 2$, throughout the paper.

We denote by $\mathcal{D}$ the family of closed dyadic cubes in $\mathbb{R}^{n}$. We let $\mathcal{D}_{j}$ be the family of those dyadic cubes whose side length is $2^{-j}, j \in \mathbb{Z}$. For a domain $G$ we fix its Whitney decomposition $W=W_{G} \subset \mathcal{D}$. For the properties of dyadic cubes and Whitney cubes we refer to Elias M. Stein's book, [S]. We write $Q^{*}=\frac{9}{8} Q$ for $Q \in W$. Then,

$$
\frac{3}{4} \operatorname{diam}(Q) \leq \operatorname{dist}(x, \partial G) \leq 6 \operatorname{diam}(Q), \quad \text { if } x \in Q^{*} .
$$

Let us fix a cube $Q_{0}$ in the Whitney decomposition $W$. For each $Q \in W$ there exists a chain of cubes $\left(Q_{0}^{*}, Q_{1}^{*}, \cdots, Q_{k}^{*}\right)=: C\left(Q^{*}\right)$ joining two cubes $Q_{0}^{*}$ and $Q_{k}^{*}=Q^{*}$ such that $Q_{i}^{*} \cap Q_{j}^{*} \neq \emptyset$ if and only if $|i-j| \leq 1$. The length of this chain is written as $\ell\left(C\left(Q^{*}\right)\right):=k$. Once the chains of cubes have been picked up, then for each Whitney cube $A$ we define a set $A(W)=\left\{Q \in W \mid A^{*} \in C\left(Q^{*}\right)\right\}$. We call this construction a chain decomposition of $G$ with a fixed cube $Q_{0}$.

The side length of a cube $Q$ in $\mathbb{R}^{n}$ is denoted by $\ell(Q)$. We write $\chi_{E}$ for the characteristic function of a set $E$. The Lebesque $n$-measure of a measurable set $E$ is denoted by $|E|$. The upper Minkowski dimension of a set $E$ in $\mathbb{R}^{n}$ is

$$
\operatorname{dim}_{\mathcal{M}}(E):=\sup \left\{\lambda \geq 0 \mid \limsup _{r \rightarrow 0+} \mathcal{M}_{\lambda}(E, r)=\infty\right\},
$$

where

$$
\mathcal{M}_{\lambda}(E, r):=\frac{\left|E+B^{n}(0, r)\right|}{r^{n-\lambda}}=\frac{\left|\cup_{x \in E} B^{n}(x, r)\right|}{r^{n-\lambda}}, \quad r>0,
$$

is the $\lambda$-dimensional Minkowski precontent.

The notation $a \lesssim b$ is used to express that an estimate $a \leq c b$ holds for some constant $c>0$ whose value is clear from the context. We use subscripts to indicate the dependence on parameters, for example, a quantity $c_{\lambda}$ depends on a parameter $\lambda$.

The following lemma gives a fractional inequality in a cube.

2.2. Lemma. Let $Q$ be a closed cube in $\mathbb{R}^{n}$. Let $1 \leq q \leq p<\infty$ and let $\delta, \rho \in(0,1)$. Then, there is a constant $c<\infty$ independent of $u \in L^{p}(Q)$ 
such that

$$
\begin{aligned}
& \frac{1}{|Q|} \int_{Q}\left|u(y)-u_{Q}\right|^{q} d y \\
& \leq c|Q|^{q(\delta / n-1 / p)}\left(\int_{Q} \int_{Q \cap B^{n}(y, \rho \ell(Q))} \frac{|u(y)-u(z)|^{p}}{|z-y|^{n+\delta p}} d z d y\right)^{q / p} .
\end{aligned}
$$

Proof. Without loss of generality we may assume that $Q=[0,1]^{n}$. This comes from a simple scaling and translation argument.

Let us divide $Q$ into $k^{n}$ congruent and closed subcubes $Q_{1}, \ldots, Q_{k^{n}}$, where $k$ is chosen such that $R \subset B^{n}(y, \rho)$ for every $y \in R$ whenever $R$ is a union of two cubes $Q_{i}$ and $Q_{j}, i, j \in\left\{1,2, \ldots, k^{n}\right\}$, sharing a common face; in particular, the case $i=j$ is allowed. We obtain

$$
\begin{aligned}
\frac{1}{|R|} \int_{R}\left|u(y)-u_{R}\right|^{q} d y & \leq\left(\frac{1}{|R|} \int_{R}\left|u(y)-u_{R}\right|^{p} d y\right)^{q / p} \\
& \leq\left(\frac{1}{|R|} \int_{R} \frac{1}{|R|} \int_{R}|u(y)-u(z)|^{p} d z d y\right)^{q / p} \\
& \lesssim|R|^{q(\delta / n-1 / p)}\left(\int_{R} \int_{R} \frac{|u(y)-u(z)|^{p}}{|z-y|^{n+\delta p}} d z d y\right)^{q / p} \\
& \lesssim\left(\int_{Q} \int_{Q \cap B^{n}(y, \rho)} \frac{|u(y)-u(z)|^{p}}{|z-y|^{n+\delta p}} d z d y\right)^{q / p} .
\end{aligned}
$$

Hölder's inequality and Minkowski's inequality yield

$$
\begin{aligned}
& \frac{1}{|Q|} \int_{Q}\left|u(y)-u_{Q}\right|^{q} d y \lesssim \frac{1}{|Q|} \int_{Q}\left|u(y)-u_{Q_{1}}\right|^{q} d y \\
& \lesssim \sum_{j=1}^{k^{n}} \int_{Q_{j}}\left|u(y)-u_{Q_{j}}\right|^{q} d y+\sum_{j=1}^{k^{n}} \int_{Q_{j}}\left|u_{Q_{j}}-u_{Q_{1}}\right|^{q} d y .
\end{aligned}
$$

By (2.3) it is enough to estimate the second series in (2.4). Let us fix $Q_{j}, j \in\left\{1, \ldots, k^{n}\right\}$, and let $\sigma:\{1,2, \ldots, k n\} \rightarrow\left\{1,2, \ldots, k^{n}\right\}$ be such that $\sigma(1)=1, \sigma(k n)=j$, and the subsequent cubes $Q_{\sigma(i)}$ and $Q_{\sigma(i+1)}$ share a common face if $i=1, \ldots, k n-1$. Since $k n \lesssim 1$, we obtain

$$
\begin{aligned}
\left|u_{Q_{j}}-u_{Q_{1}}\right|^{q} & \leq\left(\sum_{i=1}^{k n-1}\left|u_{Q_{\sigma(i+1)}}-u_{Q_{\sigma(i)}}\right|\right)^{q} \\
& \lesssim \sum_{i=1}^{k n-1}\left|u_{Q_{\sigma(i+1)}}-u_{Q_{\sigma(i+1)} \cup Q_{\sigma(i)}}\right|^{q}+\sum_{i=1}^{k n-1}\left|u_{Q_{\sigma(i+1)} \cup Q_{\sigma(i)}}-u_{Q_{\sigma(i)}}\right|^{q} .
\end{aligned}
$$


Let us consider the first sum in (2.5). Note that

$$
\begin{aligned}
& \mid u_{Q_{\sigma(i+1)}}-u_{\left.Q_{\sigma(i+1)} \cup Q_{\sigma(i)}\right|^{q}} \\
& \leq \frac{1}{\left|Q_{\sigma(i+1)}\right|} \int_{Q_{\sigma(i+1)}}\left|u_{Q_{\sigma(i+1)}}-u(y)+u(y)-u_{Q_{\sigma(i+1)} \cup Q_{\sigma(i)}}\right|^{q} d y \\
& \quad \leq \frac{1}{\left|Q_{\sigma(i+1)}\right|} \int_{Q_{\sigma(i+1)}}\left|u(y)-u_{Q_{\sigma(i+1)}}\right|^{q} d y \\
& \quad+\frac{1}{\left|Q_{\sigma(i+1)} \cup Q_{\sigma(i)}\right|} \int_{Q_{\sigma(i+1)} \cup Q_{\sigma(i)}}\left|u(y)-u_{Q_{\sigma(i+1)} \cup Q_{\sigma(i)}}\right|^{q} d y .
\end{aligned}
$$

By (2.3) we obtain

$$
\sum_{i=1}^{k n-1}\left|u_{Q_{\sigma(i+1)}}-u_{Q_{\sigma(i+1)} \cup Q_{\sigma(i)}}\right|^{q} \lesssim\left(\int_{Q} \int_{Q \cap B^{n}(y, \rho)} \frac{|u(y)-u(z)|^{p}}{|z-y|^{n+\delta p}} d z d y\right)^{q / p} .
$$

Similar estimates for the remaining sum in (2.5) conclude the proof.

We also need some estimates involving porous sets in $\mathbb{R}^{n}$.

2.6. Definition. A set $S$ in Euclidean $n$-space is porous in $\mathbb{R}^{n}$ if for some $\kappa \in(0,1]$ the following statement is true: for every $x \in \mathbb{R}^{n}$ and $0<r \leq 1$ there is $y \in B^{n}(x, r)$ such that $B^{n}(y, \kappa r) \cap S=\emptyset$.

The following lemma gives a norm estimate related to porous sets, and it is based on maximal function techniques. This lemma might be of independent interest.

2.7. Lemma. Suppose that $S$ is porous in $\mathbb{R}^{n}$ and let $1 \leq p<\infty$. If $x \in S$ and $0<r \leq 1$, then

$$
\int_{B^{n}(x, r)} \log ^{p} \frac{1}{\operatorname{dist}(y, S)} d y \leq c r^{n}\left(1+\log ^{p} r^{-1}\right)
$$

where the constant $c$ is independent of $x$ and $r$.

Proof. Let us write

$$
C_{S}=\left\{R \in \mathcal{D}: \operatorname{dist}\left(x_{R}, S\right) /(4+\sqrt{n}) \leq \ell(R) \leq 1\right\},
$$

where $x_{R}$ is the midpoint of a dyadic cube $R$. Suppose that $R \in \mathcal{D}$ is such that $\ell(R) \leq 1$ and $\operatorname{dist}(y, S) \leq 4 \ell(R)$ for some $y \in R$. Then, since

$$
\begin{aligned}
\operatorname{dist}\left(x_{R}, S\right) & \leq \operatorname{dist}\left(x_{R}, y\right)+\operatorname{dist}(y, S) \\
& \leq \sqrt{n} \ell(R)+\operatorname{dist}(y, S) \leq(4+\sqrt{n}) \ell(R)
\end{aligned}
$$

for the midpoint of $R$, we conclude that $R \in C_{S}$.

Fix $j \in \mathbb{N}_{0}$ such that $2^{-j} \leq r<2^{-j+1}$, and consider a dyadic cube $Q \in \mathcal{D}_{j}$ for which $Q \cap B^{n}(x, r) \neq \emptyset$. By covering $B^{n}(x, r)$ with such dyadic cubes it is enough to show that

$$
\left\|\log \operatorname{dist}(\cdot, S)^{-1}\right\|_{L^{p}\left(Q \cap B^{n}(x, r)\right)}^{p} \lesssim r^{n}\left(1+\log ^{p} r^{-1}\right) .
$$


By the porosity and the Lebesgue density theorem, the $n$-measure of $S$ is zero. Hence, it is enough to consider points $y \in Q \cap B^{n}(x, r) \backslash S$. Since $x \in S$,

$$
1 \leq \frac{2 \ell(Q)}{\operatorname{dist}(y, S)}
$$

Let us consider a finite sequence of dyadic cubes

$$
Q=Q_{0}(y) \supset Q_{1}(y) \supset \cdots \supset Q_{m}(y),
$$

each of them containing the point $y$ and satisfying

$$
\ell\left(Q_{i}(y)\right) / \ell\left(Q_{i+1}(y)\right)=2, \quad i=0,1, \ldots, m-1 .
$$

The last cube is chosen to satisfy

$$
\operatorname{dist}(y, S) / 4 \leq \ell\left(Q_{m}(y)\right)<\operatorname{dist}(y, S) / 2 .
$$

From (2.10) it follows that $m \geq 1$. By (2.11) and (2.10)

$$
2^{m}=\prod_{i=0}^{m-1} \frac{\ell\left(Q_{i}(y)\right)}{\ell\left(Q_{i+1}(y)\right)}=\frac{\ell\left(Q_{0}(y)\right)}{\ell\left(Q_{m}(y)\right)}>\frac{2 \ell\left(Q_{0}(y)\right)}{\operatorname{dist}(y, S)}=\frac{2 \ell(Q)}{\operatorname{dist}(y, S)} \geq 1 .
$$

Hence,

$$
m \geq \log 2^{m} \geq \log 2 \ell(Q)-\log \operatorname{dist}(y, S) \geq 0 .
$$

Furthermore, (2.12) and (2.8) yield $Q_{i}(y) \in C_{S}$ if $i=0,1, \ldots, m$. Thus, we obtain

$$
\sum_{\substack{R \in C_{S} \\ R \subset Q}} \chi_{R}(y) \geq 1+m \geq 1+\log (\ell(Q))-\log \operatorname{dist}(y, S) \geq 0,
$$

where $\chi_{R}$ is the characteristic function of $R$. Integrating this inequality and using triangle-inequality yields

$$
\begin{aligned}
& \left\|\log \operatorname{dist}(\cdot, S)^{-1}\right\|_{L^{p}\left(Q \cap B^{n}(x, r)\right)} \\
& \leq|1+\log \ell(Q)|\left|Q \cap B^{n}(x, r)\right|^{1 / p}+\left\|\sum_{\substack{R \in C_{S} \\
R \subset Q}} \chi_{R}\right\|_{L^{p}\left(\mathbb{R}^{n}\right)} .
\end{aligned}
$$

Since $S$ is porous in $\mathbb{R}^{n}$, we may follow the proof of [IV, Theorem 2.10]. We obtain a constant $K_{\kappa}$, depending on $\kappa$ in Definition 2.6, and families

$$
\{\hat{R}\}_{R \in C_{S}^{k}}, \quad C_{S}^{k} \subset C_{S}, \quad k=0,1, \ldots, K_{K}-1,
$$

where each $\{\hat{R}\}_{R \in C_{S}^{k}}$ is a disjoint family of cubes $\hat{R} \subset R$, such that

$$
\left\|\sum_{\substack{R \in C_{S} \\ R \subset Q}} \chi_{R}\right\|_{L^{p}\left(\mathbb{R}^{n}\right)} \lesssim \sum_{k=0}^{K_{K}-1}\left\|\sum_{\substack{R \in C_{S}^{k} \\ R \subset Q}} \chi_{\hat{R}}\right\|_{L^{p}\left(\mathbb{R}^{n}\right)} \leq \sum_{k=0}^{K_{K}-1}\left\|\chi_{Q}\right\|_{L^{p}\left(\mathbb{R}^{n}\right)} \lesssim|Q|^{1 / p} .
$$

By combining the estimates we obtain

$$
\left\|\log \operatorname{dist}(\cdot, S)^{-1}\right\|_{L^{p}\left(Q \cap B^{n}(x, r)\right)} \lesssim\left(1+\log \ell(Q)^{-1}\right)|Q|^{1 / p} \lesssim\left(1+\log r^{-1}\right) r^{n / p} .
$$

Estimate (2.9) follows. 


\section{Conditions for the FRactional Poincaré inequality}

In the following theorem we give sufficient conditions for a bounded domain to support the fractional $(q, p)$-Poincaré inequality (1.1).

3.1. Theorem. Let $G$ be a bounded domain in $n$-dimensional Euclidean space, $n \geq 2$, with a Whitney decomposition $W$. Let $1 \leq q \leq p<\infty$ and let $\delta, \tau \in(0,1)$.

(1) If $q<p$ and if there exists a chain decomposition of $G$ such that

$$
\sum_{A \in W}\left(\sum_{Q \in A(W)} \ell\left(C\left(Q^{*}\right)\right)^{q-1}|Q||A|^{q(\delta / n-1 / p)}\right)^{p /(p-q)}<\infty
$$

then $G$ supports the fractional (q,p)-Poincaré inequality (1.1).

(2) If $q=p$ and if there exists a chain decomposition of $G$ such that

$$
\sup _{A \in W} \sum_{Q \in A(W)} \ell\left(C\left(Q^{*}\right)\right)^{p-1}|Q||A|^{p \delta / n-1}<\infty
$$

then $G$ supports the fractional $(p, p)$-Poincaré inequality (1.1).

Proof. We prove (1); the proof of (2) is similar. Let $\delta$ and $\tau$ in $(0,1)$ be given. We use Hölder's inequality and Minkowski's inequality and then the Whitney decomposition to obtain

$$
\begin{aligned}
\int_{G}\left|u(x)-u_{G}\right|^{q} d x & \lesssim \int_{G}\left|u(x)-u_{Q_{0}^{*}}\right|^{q} d x \\
& \leq \sum_{Q \in W} \int_{Q^{*}}\left|u(x)-u_{Q_{0}^{*}}\right|^{q} d x \\
& \lesssim \sum_{Q \in W} \int_{Q^{*}}\left|u(x)-u_{Q^{*}}\right|^{q} d x+\sum_{Q \in W} \int_{Q^{*}}\left|u_{Q^{*}}-u_{Q_{0}^{*}}\right|^{q} d x .
\end{aligned}
$$

Lemma 2.2 with $\rho=2 \tau / 3$ yields

$$
\begin{aligned}
& \int_{Q^{*}}\left|u(x)-u_{Q^{*}}\right|^{q} d x \\
& \lesssim\left|Q^{*}\right|^{1+q(\delta / n-1 / p)}\left(\int_{Q^{*}} \int_{Q^{*} \cap B^{n}\left(y, \rho \ell\left(Q^{*}\right)\right)} \frac{|u(z)-u(y)|^{p}}{|z-y|^{n+\delta p}} d z d y\right)^{q / p} .
\end{aligned}
$$


Inequalities $(2.1)$ and $(1+q \delta / n-q / p)(p /(p-q))>1$ imply

$$
\begin{aligned}
& \sum_{Q \in W} \int_{Q^{*}}\left|u(x)-u_{Q^{*}}\right|^{q} d x \\
& \lesssim \sum_{Q \in W}|Q|^{1+q \delta / n-q / p}\left(\int_{Q^{*}} \int_{Q^{*} \cap B^{n}\left(y, \rho \ell\left(Q^{*}\right)\right)} \frac{|u(y)-u(z)|^{p}}{|z-y|^{n+\delta p}} d z d y\right)^{q / p} \\
& \leq\left(\sum_{Q \in W}\left(|Q|^{1+q \delta / n-q / p}\right)^{p /(p-q)}\right)^{(p-q) / p} \\
& \left(\sum_{Q \in W} \int_{Q^{*}} \int_{Q^{*} \cap B^{n}\left(y, \rho \ell\left(Q^{*}\right)\right)} \frac{|u(y)-u(z)|^{p}}{|z-y|^{n+\delta p}} d z d y\right)^{q / p} \\
& \lesssim\left(\int_{G} \int_{G \cap B^{n}(y, \tau \operatorname{dist}(y, \partial G))} \frac{|u(y)-u(z)|^{p}}{|z-y|^{n+\delta p}} d z d y\right)^{q / p} \cdot
\end{aligned}
$$

Next, we estimate the latter sum in (3.4). By using chains from the chain decomposition we obtain

$$
\begin{aligned}
\sum_{Q \in W} \int_{Q^{*}}\left|u_{Q^{*}}-u_{Q_{0}^{*}}\right|^{q} d x & \lesssim \sum_{Q \in W}|Q|\left(\sum_{j=1}^{k}\left|u_{Q_{j}^{*}}-u_{Q_{j-1}^{*}}\right|\right)^{q} \\
& \leq \sum_{Q \in W} \ell\left(C\left(Q^{*}\right)\right)^{q-1}|Q|\left(\sum_{j=1}^{k}\left|u_{Q_{j}^{*}}-u_{Q_{j-1}^{*}}\right|^{q}\right) .
\end{aligned}
$$

Estimate $\max \left\{\left|Q_{j}^{*}\right|,\left|Q_{j-1}^{*}\right|\right\} \lesssim\left|Q_{j}^{*} \cap Q_{j-1}^{*}\right|$ and Hölder's inequality yield

$$
\begin{aligned}
\left|u_{Q_{j}^{*}}-u_{Q_{j-1}^{*}}\right|^{q} & \lesssim \sum_{i=j-1}^{j}\left(\left|Q_{i}^{*}\right|^{-1} \int_{Q_{i}^{*}}\left|u(x)-u_{Q_{i}^{*}}\right| d x\right)^{q} \\
& \leq \sum_{i=j-1}^{j}\left|Q_{i}^{*}\right|^{-1} \int_{Q_{i}^{*}}\left|u(x)-u_{Q_{i}^{*}}\right|^{q} d x .
\end{aligned}
$$

Lemma 2.2 with $\rho=2 \tau / 3$ implies

$$
\begin{aligned}
& \left|u_{Q_{j}^{*}}-u_{Q_{j-1}^{*}}\right|^{q} \\
& \lesssim \sum_{i=j-1}^{j}\left|Q_{i}^{*}\right|^{q(\delta / n-1 / p)}\left(\int_{Q_{i}^{*}} \int_{Q_{i}^{*} \cap B^{n}\left(y, \rho \ell\left(Q_{i}^{*}\right)\right)} \frac{|u(z)-u(y)|^{p}}{|z-y|^{n+\delta p}} d z d y\right)^{q / p} .
\end{aligned}
$$

We have obtained for the second sum in (3.4)

$$
\begin{aligned}
& \sum_{Q \in W} \int_{Q^{*}}\left|u_{Q^{*}}-u_{Q_{0}^{*}}\right|^{q} d x \\
& \lesssim \sum_{Q \in W} \ell\left(C\left(Q^{*}\right)\right)^{q-1}|Q| \\
& \quad\left(\sum_{j=0}^{k}\left|Q_{j}^{*}\right|^{q(\delta / n-1 / p)}\left(\int_{Q_{j}^{*}} \int_{Q_{j}^{*} \cap B^{n}\left(y, \rho \ell\left(Q_{j}^{*}\right)\right.} \frac{|u(z)-u(y)|^{p}}{|z-y|^{n+\delta p}} d z d y\right)^{q / p}\right) .
\end{aligned}
$$


When we rearrange the double sum, we obtain

$$
\begin{aligned}
& \sum_{Q \in W} \int_{Q^{*}}\left|u_{Q^{*}}-u_{Q_{0}^{*}}\right|^{q} d x \\
& \lesssim \sum_{A \in W} \sum_{Q \in A(W)} \ell\left(C\left(Q^{*}\right)\right)^{q-1}|Q||A|^{q(\delta / n-1 / p)} \\
& \quad\left(\int_{A^{*}} \int_{A^{*} \cap B^{n}\left(y, \rho \ell\left(A^{*}\right)\right)} \frac{|u(y)-u(z)|^{p}}{|z-y|^{n+\delta p}} d z d y\right)^{q / p} .
\end{aligned}
$$

Hölder's inequality with $\left(\frac{p}{q}, \frac{p}{p-q}\right)$, and inequalities (3.2) and (2.1) yield

$$
\begin{aligned}
\sum_{Q \in W} \int_{Q^{*}} \mid u_{Q^{*}}-u_{\left.Q_{0}^{*}\right|^{q}} d x & \lesssim\left(\sum_{A \in W} \int_{A^{*}} \int_{A^{*} \cap B^{n}\left(y, \rho \ell\left(A^{*}\right)\right)} \frac{|u(y)-u(z)|^{p}}{|z-y|^{n+\delta p}} d z d y\right)^{q / p} \\
& \lesssim\left(\int_{G} \int_{G \cap B^{n}(y, \tau \operatorname{dist}(y, \partial G))} \frac{|u(y)-u(z)|^{p}}{|z-y|^{n+\delta p}} d z d y\right)^{q / p} .
\end{aligned}
$$

Hence, $G$ supports the fractional ( $q, p)$-Poincaré inequality (1.1).

3.5. Remark. Let $G$ be a dounded domain in $\mathbb{R}^{n}$ and let $1 \leq p<\infty$. By [Hu, Theorem 6.6] the estimate

$$
\sup _{A \in W} \sum_{Q \in A(W)} \ell\left(C\left(Q^{*}\right)\right)^{p-1}|Q||A|^{p / n-1}<\infty
$$

is a sufficient condition for the classical $(p, p)$-Poincaré inequality to be valid in the domain $G$. A comparison to our sufficient condition (3.3) for the fractional $(p, p)$-Poincaré inequality shows that condition (3.6) for the classical $(p, p)$-Poincaré inequality is weaker.

\section{Positive Results For 1-John domains}

As an application of Theorem 3.1 we show that 1-John domains support the fractional ( $p, p)$-Poincaré inequality, Theorem 4.3. We also consider fractional Sobolev-Poincaré inequalities, Theorem 4.10 and Remark 4.14. We recall that bounded uniform and Lipschitz domains are examples of 1-John domains.

4.1. Definition. A bounded domain $G$ in $\mathbb{R}^{n}, n \geq 2$, is an $s$-John domain, $s \geq 1$, if there is a point $x_{0}$ in $G$ and a constant $c>0$ such that every point $x$ in $G$ can be joined to $x_{0}$ by a rectifiable curve $\gamma:[0, l] \rightarrow G$ parametrized by its arc length for which $\gamma(0)=x, \gamma(l)=x_{0}, l \leq c$, and

$$
\operatorname{dist}(\gamma(t), \partial G) \geq t^{s} / c \quad \text { for } t \in[0, l] .
$$

The point $x_{0}$ is called an $s$-John center of $G$.

If $G$ is a 1 -John domain, then its boundary $\partial G$ is porous in $\mathbb{R}^{n}$, Definition 2.6. The boundary of an $s$-John domain with $s>1$ may have positive Lebesgue $n$-measure, $[N]$, and thus it is not necessarily porous in $\mathbb{R}^{n}$. 
Let us construct a chain decomposition of a given $s$-John domain $G$. Let $Q \in W=W_{G}$ and fix a rectifiable curve $\gamma$ that is parametrized by its arc length and joins the midpoints $x_{Q}$ and $x_{0}:=x_{Q_{0}}$, Definition 4.1. Assume that $x_{Q_{0}}$ lies in one of the cubes intersecting $Q$. Join $x_{Q}$ to $x_{Q_{0}}$ by an arc that is contained in $Q \cup Q_{0}$ and whose length is comparable to $\ell(Q)$. Otherwise there is $r>0$ such that $\gamma(r)$ lies in the boundary of a Whitney cube $P$ that intersects $Q$ and $\gamma(t)$ belongs to a cube that is not intersecting $Q$ whenever $t \in(r, \ell(\gamma)]$. Join the midpoint $x_{Q}$ to the midpoint $x_{P}$ by an arc whose length is comparable to $\ell(Q)$ and is in $Q \cup P$. We iterate these steps with $Q$ replaced by $P$, and we continue until we reach $x_{Q_{0}}$. Let $\gamma_{Q}$ be this composed curve parametrized by its arc length. It is straightforward to verify that $\ell\left(\gamma_{Q}\right) \leq c$ and

$$
t^{s} / c \leq \operatorname{dist}\left(\gamma_{Q}(t), \partial G\right) \quad \text { if } t \in\left[0, \ell\left(\gamma_{Q}\right)\right],
$$

where $c>0$ depends on the $s$-John constant of $G, s$, and $n$. Let $C\left(Q^{*}\right)$ be a chain consisting of cubes $A^{*}$ such that $A \in W$ and $x_{A} \in \gamma_{Q}\left[0, \ell\left(\gamma_{Q}\right)\right]$.

For 1-John domains we first have the following result.

4.3. Theorem. A 1-John domain $G$ in $\mathbb{R}^{n}$ supports the fractional $(p, p)$ Poincaré inequality (1.1) if $1 \leq p<\infty$ and $\tau, \delta \in(0,1)$.

Proof. We may assume that $\operatorname{diam}(G) \leq 1$. By (4.2) with $s=1$ and the fact that $\gamma_{Q}, Q \in W$, connects the midpoints of cubes in $C\left(Q^{*}\right)$,

$$
\ell\left(C\left(Q^{*}\right)\right) \leq c\left(1+\log \frac{1}{\ell(Q)}\right)
$$

where the constant $c$ is independent of $Q$. If $A \in W$, then

$$
\bigcup_{Q \in A(W)} Q \subset B^{n}\left(\omega_{A}, \min \{1, c \ell(A)\}\right),
$$

where $\omega_{A}$ is the closest point in $\partial G$ to $x_{A}$ and the constant $c>0$ is independent of $A$. By (4.4) and (4.5) we obtain

$$
\begin{aligned}
& \sum_{Q \in A(W)} \ell\left(C\left(Q^{*}\right)\right)^{p-1}|Q| \lesssim \sum_{Q \in A(W)}|Q|\left(1+\log \frac{1}{\ell(Q)}\right)^{p-1} \\
& \lesssim \sum_{Q \in A(W)}|Q|\left(1+\log ^{p} \frac{1}{\ell(Q)}\right) \lesssim \sum_{Q \in A(W)} \int_{Q}\left(1+\log ^{p} \frac{1}{\operatorname{dist}(y, \partial G)}\right) d y \\
& \leq \int_{B^{n}\left(\omega_{A}, \min \{1, c \ell(A)\}\right)}\left(1+\log ^{p} \frac{1}{\operatorname{dist}(y, \partial G)}\right) d y .
\end{aligned}
$$

Since $\partial G$ is porous in $\mathbb{R}^{n}$, Lemma 2.7 yields

$$
\sum_{Q \in A(W)} \ell\left(C\left(Q^{*}\right)\right)^{p-1}|Q| \lesssim|A|\left(1+\log ^{p} \ell(A)^{-1}\right) \lesssim|A|^{1-\delta p / n} .
$$

We have verified condition (3.3) in Theorem 3.1. Hence, the domain $G$ supports the fractional $(p, p)$-Poincaré inequality.

We state an immediate corollary of Theorem 4.3. 
4.6. Corollary. Let $G$ be a bounded domain in $\mathbb{R}^{n}, n \geq 2$, and let $1 \leq p<\infty, \delta, \tau \in(0,1)$. Then $G$ supports the fractional $(p, p)$-Poincaré inequality (1.1) if $G$ is a uniform domain or a Lipschitz domain.

It is well known [B, Theorem 5.1, Lemma 3.1] that 1-John domains support Sobolev-Poincaré inequalities: if $1 \leq p \leq q \leq n p /(n-p), p<n$, then there is $c>0$ such that, for every $u \in W^{1, p}(G)$,

$$
\left(\int_{G}\left|u(x)-u_{G}\right|^{q} d x\right)^{1 / q} \leq c\left(\int_{G}|\nabla u(x)|^{p} d x\right)^{1 / p} .
$$

We consider the corresponding fractional Sobolev-Poincaré inequalities on 1-John domains, Theorem 4.10. For the proof of this theorem we need the Riesz potentials $I_{\delta}, \delta \in(0, n)$, that are defined for suitable $f$ by

$$
I_{\delta}(f)(x)=\int_{\mathbb{R}^{n}} \frac{f(y)}{|x-y|^{n-\delta}} d y .
$$

A proof of the following theorem is in [He, Theorem 1].

4.8. Theorem. Let $0<\delta<n, 1<p<q<\infty$, and $1 / p-1 / q=\delta / n$. Then $\left\|I_{\delta}(f)\right\|_{q} \leq c\|f\|_{p}$ for a constant $c>0$ independent of $f \in L^{p}\left(\mathbb{R}^{n}\right)$.

We also need the following chaining lemma. It is a slight modification of [HK, Theorem 9.3]: we add the new condition 3 but the proof adapts to our setting, and we omit the details.

4.9. Lemma. Let $G$ in $\mathbb{R}^{n}$ be a 1-John domain whose 1-John constant is $c_{J}>1$. Fix a number $M>1$. Denote by $x_{0} \in G$ the 1 -John center of $G$, and let

$$
B_{0}:=B\left(x_{0}, \operatorname{dist}\left(x_{0}, \partial G\right) / 4 M c_{J}\right) .
$$

Then, there is a constant $c>0$, depending on $G, M$, and $n$, as follows: given $x \in G$ there is a sequence of balls $B_{i}=B\left(x_{i}, r_{i}\right) \subset G, i=0,1, \ldots$, such that for all $i=0,1, \ldots$, the following conditions $1-5$ hold:

1. $\left|B_{i} \cup B_{i+1}\right| \leq c\left|B_{i} \cap B_{i+1}\right|$;

2. $\operatorname{dist}\left(x, B_{i}\right) \leq c r_{i}$;

3. $\operatorname{dist}\left(B_{i}, \partial G\right) \geq M r_{i}$;

4. $\left|x-x_{i}\right| \leq c r_{i}$ and $r_{j} \rightarrow 0$ as $j \rightarrow \infty$;

5. $\sum_{j=0}^{\infty} \chi_{B_{j}} \leq c \chi_{G}$.

The following result is a fractional Sobolev-Poincaré inequality for 1-John domains.

4.10. Theorem. Assume that $G$ is a 1-John domain in $\mathbb{R}^{n}, n \geq 2$. Suppose that $\tau, \delta \in(0,1), p<n / \delta$, and

$$
1<p \leq q \leq \frac{n p}{n-\delta p} .
$$

Then $G$ supports the fractional $(q, p)$-Poincaré inequality (1.1). 
Proof. By Hölder's inequality we may assume that $q=n p /(n-\delta p)$. Fix $\tau \in(0,1)$ and let $u \in L^{p}(G)$. Let $x \in G$ be a Lebesgue point of $u$, and consider the associated balls $B_{i}=B\left(x_{i}, r_{i}\right)$ from Lemma 4.9 satisfying conditions $1-5$ with $M>2 / \tau$.

The following holds: for all $i$,

$$
B_{i} \subset B^{n}(y, \tau \operatorname{dist}(y, \partial G)), \quad \text { if } y \in B_{i} .
$$

Namely, let us fix $y \in B_{i}$ and let $z$ be any point in $B_{i}$. Then, by condition 3 in Lemma 4.9,

$$
\begin{aligned}
|z-y| & \leq\left|y-x_{i}\right|+\left|x_{i}-z\right| \leq 2 r_{i} \leq 2 \frac{\operatorname{dist}\left(B_{i}, \partial G\right)}{M} \\
& \leq \frac{2}{M} \operatorname{dist}(y, \partial G)<\tau \operatorname{dist}(y, \partial G) .
\end{aligned}
$$

By the Lebesgue differentiation theorem and condition 4 in Lemma 4.9,

$$
u(x)=\lim _{i \rightarrow \infty} \frac{1}{\left|B_{i}\right|} \int_{B_{i}} u(y) d y=\lim _{i \rightarrow \infty} u_{B_{i}} .
$$

Hence, by condition 1 in Lemma 4.9, we obtain

$$
\begin{aligned}
\left|u(x)-u_{B_{0}}\right| & \leq \sum_{i=0}^{\infty}\left|u_{B_{i}}-u_{B_{i+1}}\right| \\
& \leq \sum_{i=0}^{\infty}\left(\left|u_{B_{i}}-u_{B_{i} \cap B_{i+1}}\right|+\left|u_{B_{i+1}}-u_{B_{i} \cap B_{i+1}}\right|\right) \\
& \lesssim \sum_{i=0}^{\infty} \frac{1}{\left|B_{i}\right|} \int_{B_{i}}\left|u(y)-u_{B_{i}}\right| d y .
\end{aligned}
$$

For a ball $B_{i}$,

$$
\begin{aligned}
\frac{1}{\left|B_{i}\right|} \int_{B_{i}}\left|u(y)-u_{B_{i}}\right| d y & =\frac{1}{\left|B_{i}\right|} \int_{B_{i}}\left|\frac{1}{\left|B_{i}\right|} \int_{B_{i}}(u(y)-u(z)) d z\right| d y \\
& \leq \frac{1}{\left|B_{i}\right|} \int_{B_{i}}\left(\frac{1}{\left|B_{i}\right|} \int_{B_{i}}|u(y)-u(z)|^{p} d z\right)^{1 / p} d y \\
& =\frac{1}{\left|B_{i}\right|^{1+1 / p}} \int_{B_{i}}\left(\int_{B_{i}}|u(y)-u(z)|^{p} d z\right)^{1 / p} d y \\
& \lesssim\left|B_{i}\right|^{\delta / n-1} \int_{B_{i}}\left(\int_{B_{i}} \frac{|u(y)-u(z)|^{p}}{|y-z|^{n+\delta p}} d z\right)^{1 / p} d y
\end{aligned}
$$

Let us write

$$
g(y):=\left(\int_{G \cap B^{n}(y, \tau \operatorname{dist}(y, \partial G))} \frac{|u(y)-u(z)|^{p}}{|y-z|^{n+\delta p}} d z\right)^{1 / p} .
$$


By (4.12), (4.11) and condition 2 in Lemma 4.9,

$$
\begin{aligned}
\sum_{i=0}^{\infty} \frac{1}{\left|B_{i}\right|} \int_{B_{i}}\left|u(y)-u_{B_{i}}\right| d y & \lesssim \sum_{i=0}^{\infty}\left|B_{i}\right|^{\delta / n-1} \int_{B_{i}}\left(\int_{B_{i}} \frac{|u(y)-u(z)|^{p}}{|y-z|^{n+\delta p}} d z\right)^{1 / p} d y \\
& \leq \sum_{i=0}^{\infty}\left|B_{i}\right|^{\delta / n-1} \int_{B_{i}}\left(\int_{B^{n}(y, \tau \operatorname{dist}(y, \partial G))} \frac{|u(y)-u(z)|^{p}}{|y-z|^{n+\delta p}} d z\right)^{1 / p} d y \\
& \lesssim \sum_{i=0}^{\infty} r_{i}^{n(\delta / n-1)} \int_{B_{i}} g(y) d y \\
& \lesssim \sum_{i=0}^{\infty} \int_{B_{i}} \frac{g(y)}{|x-y|^{n-\delta}} d y .
\end{aligned}
$$

By condition 5 in Lemma 4.9,

$$
\left|u(x)-u_{B_{0}}\right| \lesssim \int_{G} \frac{g(y)}{|x-y|^{n-\delta}} d y=I_{\delta}\left(\chi_{G} g\right)(x)
$$

for every Lebesgue point $x \in G$. By integrating this inequality and using Theorem 4.8, we obtain

$$
\begin{aligned}
\left(\int_{G}\left|u(x)-u_{B_{0}}\right|^{q} d x\right)^{1 / q} & \lesssim\left\|I_{\delta}\left(\chi_{G} g\right)\right\|_{q} \lesssim\left\|\chi_{G} g\right\|_{p} \\
& =\left(\int_{G} \int_{G \cap B^{n}(y, \tau \operatorname{dist}(y, \partial G))} \frac{|u(y)-u(z)|^{p}}{|y-z|^{n+\delta p}} d z d y\right)^{1 / p} .
\end{aligned}
$$

Inequality (1.1) follows.

4.14. Remark. The proof of Theorem 4.10 also gives the following result: Suppose that $G$ is a 1 -John domain in $\mathbb{R}^{n}$. Let $\tau, \delta \in(0,1)$ and let $p, q \in[1, \infty)$ be such that

$$
0 \leq 1 / p-1 / q<\delta / n .
$$

Then $G$ supports the fractional ( $q, p$ )-Poincaré inequality (1.1). Indeed, it suffices to recall that the linear operator $f \mapsto I_{\delta}\left(\chi_{G} f\right)$ is bounded from $L^{p}(G)$ to $L^{q}(G)$, [GT, Lemma 7.12].

\section{Positive Results for $s$-John Domains With $s>1$}

We prove the fractional $(1, p)$-Poincaré inequality (1.1) for $s$-John domains, Theorem 5.1. We show in Section 6 that this result is sharp in terms of the restriction on $p$, Theorem 6.9.

5.1. Theorem. Let $s>1,1<p<\infty, \lambda \in[n-1, n)$, and let $\delta, \tau \in(0,1)$. Suppose that

$$
s<\frac{n+1-\lambda}{1-\delta}, \quad p>\frac{s(n-1)-\lambda+1}{n-s(1-\delta)-\lambda+1} .
$$

Let $G$ be an $s$-John domain in $\mathbb{R}^{n}$ such that $\operatorname{dim}_{\mathcal{M}}(\partial G) \leq \lambda$. Then $G$ supports the fractional $(1, p)$-Poincaré inequality (1.1). 
We need preparations for the proof of Theorem 5.1.

By scaling we may assume that $\operatorname{diam}(G) \leq 1$. Hence, the side lengths of all Whitney cubes in $W=W_{G}$ are bounded by one and

$$
W=\bigcup_{j=0}^{\infty} W_{j}
$$

where each $W_{j}$ stands for the family of cubes $A \in W$ with $\ell(A)=2^{-j}$.

For a given $s$-John domain $G$, we consider its chain decomposition that is constructed in Section 4. Given $j, k \in \mathbb{N}$ and $\sigma \geq 1$ we define

$$
W_{j, k, \sigma}:=\left\{A \in W_{j}\left|2^{-(j-k) n} \leq\right| \cup A(W) \mid \leq \sigma \cdot 2^{-(j-k-1) n}\right\} .
$$

The following lemma from [HH-SV, Lemma 4.7] gives the properties we need for this chain decomposition of $G$.

The integer part of $\alpha \in \mathbb{R}$ is denoted by $[\alpha]$.

5.4. Lemma. Let $s>1$ and let $G$ be an $s$-John domain in $\mathbb{R}^{n}$ such that $\operatorname{diam}(G) \leq 1$ and $\operatorname{dim}_{\mathcal{M}}(\partial G)<\lambda \in[n-1, n)$. Then, there is a constant $\sigma \geq 1$ such that

$$
W_{j}=\bigcup_{k=0}^{[j-j / s]} W_{j, k, \sigma} \quad \text { for every } j \in \mathbb{N} .
$$

Furthermore, if $k \in\{0,1, \ldots,[j-j / s]\}$, then

$$
\sharp W_{j, k, \sigma} \leq c 2^{-k n} 2^{j(n+1+(\lambda-n-1) / s)} .
$$

The positive constant $c$ depends on $n, s, \partial G$, and the $s$-John constant of the domain $G$.

We are ready for the proof of Theorem 5.1.

Proof of Theorem 5.1. Choose $\lambda^{\prime} \in(\lambda, n)$ such that (5.2) is true if $\lambda$ is replaced by $\lambda^{\prime}$. Then $\operatorname{dim}_{\mathcal{M}}(\partial G)<\lambda^{\prime}$ and hence we may assume that $\operatorname{dim}_{\mathcal{M}}(\partial G)$ is strictly less than $\lambda \in[n-1, n)$.

By Theorem 3.1 it is enough to prove the finiteness of

$$
\Sigma:=\sum_{A \in W}\left(\sum_{Q \in A(W)}|Q||A|^{\delta / n-1 / p}\right)^{p /(p-1)}=\sum_{A \in W}\left(|\cup A(W)||A|^{\delta / n-1 / p}\right)^{p /(p-1)},
$$

where the chain decomposition of $G$ is given by Lemma 5.4. By (5.3) and (5.5) in Lemma 5.4

$$
\Sigma=\sum_{j=0}^{\infty} \sum_{k=0}^{[j-j / s]} \sum_{A \in W_{j, k, \sigma}}\left(|\cup A(W)||A|^{\delta / n-1 / p}\right)^{p /(p-1)} .
$$


Then, by using the definition of $W_{j, k, \sigma}$ and (5.6) from Lemma 5.4 we obtain the estimate

$$
\begin{aligned}
\Sigma & \lesssim \sum_{j=0}^{\infty} \sum_{k=0}^{[j-j / s]} 2^{-k n} 2^{j(n+1+(\lambda-n-1) / s)} \cdot\left(2^{-(j-k) n} \cdot 2^{-j n(\delta / n-1 / p)}\right)^{p /(p-1)} \\
& =\sum_{j=0}^{\infty} \sum_{k=0}^{[j-j / s]} 2^{k n(p /(p-1)-1)} 2^{j(n+1+(\lambda-n-1) / s-n p /(p-1)-\delta p /(p-1)+n /(p-1))} .
\end{aligned}
$$

Let us fix $j$ and $k$ as in the summation above. Then,

$$
k n\left(\frac{p}{p-1}-1\right) \leq n(j-j / s)\left(\frac{p}{p-1}-1\right)=\frac{j n(1-1 / s)}{p-1} .
$$

The trivial estimate $[j-j / s] \leq j$ implies that

$$
\begin{aligned}
\Sigma & \lesssim \sum_{j=0}^{\infty} j \cdot 2^{j(n(1-1 / s) /(p-1)+n+1+(\lambda-n-1) / s-n p /(p-1)-\delta p /(p-1)+n /(p-1))} \\
& =\sum_{j=0}^{\infty} j \cdot 2^{j(n s-s+\lambda p-\lambda-n p-p+1-p(\delta-1) s) / s(p-1)} .
\end{aligned}
$$

By (5.2) the last series converges.

\section{Sharpness of TheOrem 5.1}

We show that Theorem 5.1 is sharp by proving Theorem 6.9. For this purpose we construct $s$-John domains which do not support the fractional $(1, p)$-Poincaré inequality (1.1) for certain values of $p$.

Let us recall the construction of the $s$-version of a given 1-John domain $G$, [HH-SV]. We may assume that the diameter of $G$ is restricted by condition

$$
(\ell(Q) / 8)^{s} \leq \ell(Q) / 32, \quad \text { if } Q \in W_{G} .
$$

Let $Q$ be a closed cube in $\mathbb{R}^{n}$ centered at $x=\left(x_{1}, \ldots, x_{n}\right)$ and whose side length $\ell=\ell(Q)$ satisfies $(\ell / 8)^{s} \leq \ell / 32$. Thus, $Q=\prod_{i=1}^{n}\left[x_{i}-\ell / 2, x_{i}+\ell / 2\right]$. The room in $Q$ is the open cube

$$
R(Q):=\operatorname{int}\left(\frac{1}{4} Q\right)=\prod_{i=1}^{n}\left(x_{i}-\ell / 8, x_{i}+\ell / 8\right)
$$

centered at $x$ with side length $\ell / 4$. The $s$-passage in $Q$ is the open set

$$
P_{s}(Q):=\left(\prod_{i=1}^{n-1}\left(x_{i}-(\ell / 8)^{s}, x_{i}+(\ell / 8)^{s}\right)\right) \times\left(x_{n}+\ell / 8, x_{n}+\ell / 4\right) .
$$

Since $(\ell / 8)^{s}<\ell / 8$, we have $P_{s}(Q) \subset \frac{1}{2} Q$. The long $s$-passage in $Q$ is the open set

$$
L_{s}(Q):=\left(\prod_{i=1}^{n-1}\left(x_{i}-(\ell / 8)^{s}, x_{i}+(\ell / 8)^{s}\right)\right) \times\left(x_{n}, x_{n}+\ell / 2\right) \subset Q .
$$


The $s$-apartment in $Q$ is the set

$$
A_{s}(Q):=L_{s}(Q) \cup\left(Q \backslash\left(\partial R(Q) \cup \partial P_{s}(Q)\right)\right) \subset Q .
$$

6.3. Definition. Let $G$ in $\mathbb{R}^{n}$ be a 1-John domain and let $s>1$ be a number such that (6.1) holds. Then, the s-version of $G$ is the domain

$$
G_{s}:=Q_{0} \cup \bigcup_{\substack{Q \in W_{G} \\ Q \neq Q_{0}}} A_{s}(Q) .
$$

Here $Q_{0} \in W_{G}$ is the cube containing the 1-John center $x_{0}$ of $G$.

We construct test functions. Let $Q \in W_{G}$ be fixed, and define the tiny s-passage in $Q$ to be the open set

$$
T_{s}(Q):=\left(\prod_{i=1}^{n-1}\left(x_{i}-(\ell / 8)^{s}, x_{i}+(\ell / 8)^{s}\right)\right) \times\left(x_{n}+5 \ell / 32, x_{n}+7 \ell / 32\right) .
$$

Then, we define a continuous function

$$
u^{A_{s}(Q)}: G_{s} \rightarrow \mathbb{R}
$$

which has linear decay along the $n^{\text {th }}$ variable in $T_{s}(Q)$ and is constant in both components of $P_{s}(Q) \backslash T_{s}(Q)$, and satisfies

$$
u^{A_{s}(Q)}(x)= \begin{cases}\ell(Q)^{(\lambda-n) / q}, & \text { if } x \in R(Q) ; \\ 0, & \text { if } x \in G_{s} \backslash\left(R(Q) \cup P_{s}(Q)\right) .\end{cases}
$$

In the sense of distributions in $G_{s}$,

$$
\nabla u^{A_{s}(Q)}=\left(0, \ldots, 0,-16 \ell(Q)^{(\lambda-n) / q-1} \chi_{T_{s}(Q)}\right)
$$

pointwise almost everywhere.

The reason why we do not let $u^{A_{s}(Q)}$ have linear decay along the whole $s$-passage $P_{s}(Q)$ is that we need the following property.

6.6. Remark. Let $Q \in W_{G}$. Suppose that $x \in G_{s}$ and $y \in B^{n}\left(x, \operatorname{dist}\left(x, \partial G_{s}\right)\right)$ are such that

$$
\left|u^{A_{s}(Q)}(x)-u^{A_{s}(Q)}(y)\right| \neq 0 .
$$

Then $x$ and $y$ both belong to $P_{s}(Q)$. This fact follows from the assumption (6.1).

The following proposition is the main tool for proving Theorem 6.9.

6.7. Proposition. Let $G$ be a 1-John domain in $\mathbb{R}^{n}$ and $s>1$ be such that (6.1) holds. Suppose that

$$
\limsup _{k \rightarrow \infty} 2^{-\lambda k} \cdot \sharp W_{k}>0, \quad \text { where } \lambda=\operatorname{dim}_{\mathcal{M}}(\partial G) \in[n-1, n) \text {. }
$$

Let $\delta, \tau \in(0,1)$ and $1 \leq q<p<\infty$ be such that

$$
\frac{(p-q)(\lambda-n)}{p q}+\frac{(s-1)(n-1)}{p} \geq 1-s(1-\delta) .
$$

Then the s-version of $G$ is an $s$-John domain with $\operatorname{dim}_{\mathcal{M}}\left(\partial G_{s}\right)=\lambda$ and $G_{s}$ does not support the fractional $(q, p)$-Poincaré inequality (1.1). 
Proof. The fact

$$
\operatorname{dim}_{\mathcal{M}}\left(\partial G_{s}\right)=\operatorname{dim}_{\mathcal{M}}(\partial G)=\lambda
$$

is from [HH-SV, Proposition 5.11]. By [HH-SV, Proposition 5.16], the domain $G_{s}$ is an $s$-John domain. Hence, it remains to prove the failure of the fractional Poincaré inequality.

Let us choose $k_{0} \in \mathbb{N}$ such that

$$
\limsup _{k \rightarrow \infty} 2^{-\lambda\left(k-k_{0}\right)} \cdot \sharp W_{k}>2 .
$$

This allows us to choose indices $j(k), k \in \mathbb{N}$, inductively such that

$$
\max \left\{k_{0},-\log _{2} \ell\left(Q_{0}\right)\right\}<j(1)<j(2)<\cdots
$$

and $\sharp W_{j(k)} \geq 2 \cdot 2^{\lambda\left(j(k)-k_{0}\right)}$ for every $k \in \mathbb{N}$. Let us write $M_{j}:=2^{\left[\lambda\left(j-k_{0}\right)\right]}$, where $\left[\lambda\left(j-k_{0}\right)\right]$ means the integer part of $\lambda\left(j-k_{0}\right)$, and let us choose cubes

$$
Q_{j(k)}^{1}, \ldots, Q_{j(k)}^{2 M_{j(k)}} \in W_{j(k)} \backslash\left\{Q_{0}\right\} .
$$

For every $m \in \mathbb{N}$ we define

$$
v_{m}:=\sum_{k=1}^{m}\left(\sum_{i=1}^{M_{j(k)}} u^{A_{s}\left(Q_{j(k)}^{i}\right)}-\sum_{i=M_{j(k)}+1}^{2 M_{j(k)}} u^{A_{s}\left(Q_{j(k)}^{i}\right)}\right) .
$$

Note that $\left(v_{m}\right)_{G_{s}}=0$ and

$$
\begin{aligned}
A_{m}: & =\left(\int_{G_{s}}\left|v_{m}-\left(v_{m}\right)_{G_{s}}\right|^{q}\right)^{1 / q}=\left(\sum_{k=1}^{m} \sum_{i=1}^{2 M_{j(k)}} \int_{G_{s}}\left|u^{A_{s}\left(Q_{j(k)}^{i}\right)}\right|^{q}\right)^{1 / q} \\
& \geq\left(m \cdot 2 \cdot 2^{\lambda\left(j(k)-k_{0}\right)-1} \cdot 2^{-j(k)(\lambda-n)} \cdot 4^{-n} \cdot 2^{-j(k) n}\right)^{1 / q}=c_{n, q, \lambda, k_{0}} m^{1 / q} .
\end{aligned}
$$

Next we estimate the right hand side of (1.1) with $u=v_{m}$. We write

$$
G_{s}(x):=B^{n}\left(x, \operatorname{dist}\left(x, \partial G_{s}\right)\right) \subset G_{s} \quad \text { for } x \in G_{s} .
$$

Remark 6.6 yields: if $x \in G_{s}$ and $y \in G_{s}(x)$ are such that $\left|v_{m}(x)-v_{m}(y)\right| \neq$ 0 , then $x, y \in P_{s}(Q)$ for some Whitney cube $Q \in W_{G}$. By using this we obtain

$$
\begin{aligned}
B_{m}: & =\left(\int_{G_{s}} \int_{G_{s}(x)} \frac{\left|v_{m}(x)-v_{m}(y)\right|^{p}}{|x-y|^{n+\delta p}} d y d x\right)^{1 / p} \\
& =\left(\sum_{Q \in W_{G}} \int_{Q \cap G_{s}} \int_{G_{s}(x)} \frac{\left|v_{m}(x)-v_{m}(y)\right|^{p}}{|x-y|^{n+\delta p}} d y d x\right)^{1 / p} \\
& =\left(\sum_{Q \in W_{G}} \int_{P_{s}(Q)} \int_{P_{s}(Q) \cap G_{s}(x)} \frac{\left|v_{m}(x)-v_{m}(y)\right|^{p}}{|x-y|^{n+\delta p}} d y d x\right)^{1 / p} \\
& =\left(\sum_{k=1}^{m} \sum_{i=1}^{2 M_{j(k)}} \int_{P_{s}\left(Q_{j(k)}^{i}\right)} \int_{P_{s}\left(Q_{j(k)}^{i}\right) \cap G_{s}(x)} \frac{\left|u^{A_{s}\left(Q_{j(k)}^{i}\right)}(x)-u^{A_{s}\left(Q_{j(k)}^{i}\right)}(y)\right|^{p}}{|x-y|^{n+\delta p}} d y d x\right)^{1 / p} .
\end{aligned}
$$


Let us fix a cube $R=Q_{j(k)}^{i}$, where $k \in\{1, \ldots, m\}$ and $i \in\left\{1,2, \ldots, 2 M_{j(k)}\right\}$. By $(6.5)$

$$
\left|u^{A_{s}(R)}(x)-u^{A_{s}(R)}(y)\right| \leq 16 \ell(R)^{(\lambda-n) / q-1}|x-y|, \quad x, y \in P_{s}(R) .
$$

Hence,

$$
\begin{aligned}
\mathcal{I}_{R}:= & \int_{P_{s}(R)} \int_{P_{s}(R) \cap G_{s}(x)} \frac{\left|u^{A_{s}(R)}(x)-u^{A_{s}(R)}(y)\right|^{p}}{|x-y|^{n+\delta p}} d y d x \\
& \lesssim \ell(R)^{p(\lambda-n) / q-p} \int_{P_{s}(R)} \int_{P_{s}(R) \cap G_{s}(x)}|x-y|^{-n+(1-\delta) p} d y d x .
\end{aligned}
$$

Note that $G_{s}(x) \subset B^{n}\left(x, \ell(R)^{s}\right)$ if $x \in P_{s}(R)$. Thus,

$$
\int_{P_{s}(R) \cap G_{s}(x)}|x-y|^{-n+(1-\delta) p} d y \leq \int_{B^{n}\left(0, \ell(R)^{s}\right)}|y|^{-n+(1-\delta) p} d y \lesssim \ell(R)^{s(1-\delta) p},
$$

and it follows that

$$
\begin{aligned}
\mathcal{I}_{R} & \lesssim \ell(R)^{p(\lambda-n) / q-p}\left|P_{s}(R)\right| \ell(R)^{s(1-\delta) p} \\
& =\ell(R)^{p(\lambda-n) / q-p+s(n-1)+1+s(1-\delta) p}=2^{-j(k)(p(\lambda-n) / q-p+s(n-1)+1+s(1-\delta) p)} .
\end{aligned}
$$

These estimates and inequality (6.8) yield

$$
B_{m} \lesssim\left(\sum_{k=1}^{m} 2^{\lambda j(k)} 2^{-j(k)(p(\lambda-n) / q-p+s(n-1)+1+s(1-\delta) p)}\right)^{1 / p} \lesssim m^{1 / p} .
$$

By using the assumption $q<p$ we obtain

$$
\frac{A_{m}}{B_{m}} \geq c_{n, s, p, q, k_{0}, \lambda, \delta} m^{1 / q-1 / p} \stackrel{m \rightarrow \infty}{\longrightarrow} \infty .
$$

Hence, the domain $G_{s}$ does not support the fractional $(q, p)$-Poincaré inequality (1.1) for any $\tau \in(0,1)$.

The following theorem shows the sharpness of Theorem 5.1.

6.9. Theorem. Let $s>1, p \in(1, \infty), \lambda \in[n-1, n)$, and let $\delta, \tau \in(0,1)$. Suppose that

$$
s<\frac{n+1-\lambda}{1-\delta}, \quad p \leq \frac{s(n-1)-\lambda+1}{n-s(1-\delta)-\lambda+1} .
$$

Then, there is an $s$-John domain $G_{s}$ in $\mathbb{R}^{n}$ with the following properties: $\operatorname{dim}_{\mathcal{M}}\left(\partial G_{s}\right)=\lambda$ and $G_{s}$ does not support the fractional $(1, p)$-Poincaré inequality (1.1).

Proof. By [HH-SV, Proposition 5.2] there is a 1-John domain $G$ in $\mathbb{R}^{n}$ such that $\operatorname{dim}_{\mathcal{M}}(\partial G)=\lambda$ and $\lim \sup _{k \rightarrow \infty} 2^{-\lambda k} \cdot \sharp W_{k}>0$. By scaling we may also assume that (6.1) holds. Hence, by Proposition 6.7 the $s$-version $G_{s}$ has required properties. 


\section{REFERENCES}

[A] Robert A. Adams, Sobolev spaces, Academic Press, Inc., Orlando, Florida, 1975.

[B] Bogdan Bojarski, Remarks on Sobolev imbedding inequalities, Complex Analysis Joensuu 1987, Lecture Notes in Math., vol. 1351, Springer, 1988, $52-68$.

[BBM1] Jean Bourgain, Haïm Brezis and Petru Mironescu, Another look at Sobolev spaces, Optimal Control and Partial Differential Equations, edited by J. L. Menaldi, E. Rofman, and A. Sulem, IOS Press, 439-455 (2001).

[BBM2] Jean Bourgain, Haïm Brezis and Petru Mironescu, Limiting embedding theorems for $W^{s, p}$ when $s \uparrow 1$ and applications, J. Anal. Math., 87 (2002), $77-101$.

[GT] David Gilbarg and Neil S. Trudinger, Elliptic partial differential equations of second order, Classics in Mathematics, Springer-Verlag, Berlin, 2001.

[HK] Piotr Hajłaz and Pekka Koskela, Sobolev met Poincaré, Memoirs Amer. Math. Soc., 688 (2000), 1-101.

[HH-SV] Petteri Harjulehto, Ritva Hurri-Syrjänen and Antti V. Vähäkangas, On the $(1, p)$-Poincaré inequality, University of Helsinki, Department of Mathematics and Statistics Report series, 519 (2011).

[He] Lars Inge Hedberg, On certain convolution inequalities, Proc. Amer. Math. Soc., 36 (1972), 505-510.

[Hu] Ritva Hurri, Poincaré domains in $\mathbb{R}^{n}$, Ann. Acad. Sci. Fenn. Ser. A Math. Dissertationes, 71 (1988).

[IV] Lizaveta Ihnatsyeva and Antti V. Vähäkangas, Characterization of traces of smooth functions on Ahlfors regular sets, arXiv:1109.2248 (2011).

[MS1] Vladimir Maz'ya and Tatyana Shaposhnikova, On the Bourgain, Brezis, and Mironescu theorem concerning limiting embeddings of fractional Sobolev spaces, J. Funct. Anal., 195 (2002), 230-238.

[MS2] Vladimir Maz'ya and Tatyana Shaposhnikova, Erratum to "On the Bourgain, Brezis, and Mironescu theorem concerning limiting embeddings of fractional Sobolev spaces", J. Funct. Anal., 201 (2003), 298-300.

[N] Tomi Nieminen, Generalized mean porosity and dimension, Ann. Acad. Sci. Fenn. Math. Ser. A I Math., 31 (2006), 143-172.

[P1] Augusto C. Ponce, A variant of Poincaré inequality, C. R. Acad. Sci. Paris Ser. I, 337 (2003), 253-257.

[P2] Augusto C. Ponce, An estimate in the spirit of Poincaré's inequality, J. Eur. Math. Soc., 6 (2004), 1-15.

[S] Elias M. Stein, Singular integrals and differentiability properties of functions, Princeton Univ. Press, Princeton, New Jersey, 1970.

Department of Mathematics and Statistics, Gustaf Hällströmin Katu 2b, FI-00014 University of Helsinki, Finland

E-mail address: ritva.hurri-syrjanen@helsinki.fi

E-mail address: antti.vahakangas@helsinki.fi 